Li et al., Afr J Tradit Complement Altern Med. (2013) 10(4):179-185

http://dx.doi.org/10.4314/ajtcam.v10i4.29

\title{
APPLIED ORTHOGONAL EXPERIMENT DESIGN FOR THE OPTIMUM \\ MICROWAVE-ASSISTED EXTRACTION CONDITIONS OF POLYSACCHARIDES FROM RHODIOLAE RADIX
}

\author{
Fenglin Li ${ }^{1,2,3}{ }^{*}$, Furen Xiao ${ }^{3,}$ Jingli Gong ${ }^{1,2}$, Tian $\mathrm{Yu}^{1}$ \\ ${ }^{1}$ Jilin Agricultural Science and Technology College, Jilin, 132101, China, ${ }^{2}$ Brewing Technology \\ Innovation Center of Jilin Province, Jilin, 132101, China, ${ }^{3}$ Key Laboratory of Metastable Materials \\ Science and Technology, College of Materials Science and Engineering, Yanshan University, \\ Qinghuangdao, 066004, China. \\ *E-mail: swgclfl@163.com; swgclifenglin@sina.com
}

\begin{abstract}
An experiment on polysaccharides from Rhodiolae Radix (PRR) extraction was carried out using microwave-assisted extraction (MAE) method with an objective to establishing the optimum MAE conditions of PRR. Single factor experiments were performed to determine the appropriate range of extraction conditions, and the optimum conditions were obtained using orthogonal experiment design. The results showed that the optimum MAE conditions of PRR were as follows: solid-liquid ratio of 1:45 g/mL, irradiation power of $480 \mathrm{~W}$, and irradiation time of $8 \mathrm{~min}$, while extraction yield of PRR was $3.24 \%$.
\end{abstract}

Keywords: Rhodiolae Radix; polysaccharides; orthogonal experiment design; microwave-assisted extraction

\section{Introduction}

Rhodiola sachalinensis A. BOR, a perennial herbaceous plant, belongs to the family Crassulaceae, and the root of the plant (Gao-shan-hong-jing-tian in Chinese), Rhodiolae Radix, has been widely used in traditional Chinese medicine for more than 800 years (Wu et al., 2003; Yan et al., 2004). As a drug that enhances the body "source of adaptation to environment", Rhodiolae Radix has been used in such special occupations as diving, astronomy, piloting and mountaineering to enhance the body's ability to survive in adverse environments (Mao et al., 2007; Wiedenfeld et al., 2007). Current researches indicate that Rhodiolae Radix has many biological activities, such as adaptogenic, antihypoxia, antioxidant, anti-inflammatory, antiviral, antidiabetic, anticancer, immune enhancing and sexually stimulating properties (Choe et al., 2012; Li et al., 2011; Su et al., 2007; Zhang et al., 2007). Rhodiolae Radix is reported to contain some biologically active components, such as phenylethanol derivatives (rhodioloside or salidioside, tyrosol), flavones (quercetin, hyperoside, kaempferol, rhodiolin, rhodionin, tricin, acetylrodalgin, catechins and proanthocyanins), phenylpropanoids (rosavin, rosin and rosarin), polysaccharides and the others, including phenolic acids, ethyl gallate, ethereal oil, organic acids and lipids (Wiedenfeld et al., 2007). To date, its biological activities are mainly attributed to salidroside and the phenylpropanoids. However, recent studies have suggested that the polysaccharides from Rhodiolae Radix (PRR) also exhibit significant biological activities, which include antiviral, antidiabetic, and immune enhancing functions, and also the promotion of the recovery of hematopoietic function in myelosuppressed (Li et al., 2006; Piao et al., 2000; Cheng et al., 1993; Zhang and Liu, 2009).

Extraction of polysaccharides is an important step for its application or further research and development. Conventional techniques for solvent extraction of polysaccharides usually require long extraction times, and extraction efficiency is low 


\section{http://dx.doi.org/10.4314/ajtcam.v10i4.29}

(Wu et al., 2001). Recently some new extraction methods have been developed to improve the extraction process, such as supercritical carbon dioxide extraction (SFE), supercritical or subcritical water extraction, microwave-assisted extraction (MAE) and ultrasound-assisted extraction (UAE). Among these, MAE is based on absorption of microwave energy by molecules of polar chemical compounds and can heat the material homogeneously (Romanik et al., 2007; Wang et al., 2009). Compared with conventional solvent extraction, MAE method has many advantages, such as shorter time, less solvent, higher extraction rate, lower cost and better products, without changing the molecular structure (Xie et al., 2010). Nevertheless, few studies have focused on the optimum MAE conditions of polysaccharides from Rhodiolae Radix (PRR). The aim of the present work was to establish the optimum UAE conditions of PRR by using orthogonal experiment design in order to achieve scientific evidence for the processing and utilising of Rhodiolae Radix.

\section{Materials and methods}

\section{Plant materials}

Rhodiolae Radix was purchased from a local drug market and the material was identified by Mr. Wang Guang Yao, a botanist of Jilin Agriculture Science and Technology College. A voucher specimen (No. 0429161) has been deposited in herbarium of Jilin Agriculture Science and Technology College (Jilin, China). The dried Rhodiolae Radix was ground in a high speed disintegrator (Model HDV, Dongying Hongjiu Traditional Medicine Machine Company, Shandong, China) to obtain a fine powder (particle diameter size: $1-2 \mathrm{~mm}$ ). The powder was stored at $4{ }^{\circ} \mathrm{C}$ until use.

\section{Chemicals and reagents}

Glucose $\left(\mathrm{C}_{6} \mathrm{H}_{12} \mathrm{O}_{6}\right)$ (AR class) was obtained from the National Institutes for Food and Drug Control (Beijing, China). All other chemicals and reagents used were of analytical grade. Water was purified with a Milli-Q purification system and was used to prepare all solutions.

\section{Extraction procedure}

Microwave-assisted extraction (MAE) was performed with a commercial WD800ASL microwave extraction apparatus (Galanz Co., Shunde, China). The apparatus was a rectangular container $(510 \times 350 \times 300 \mathrm{~mm})$ and the power rating was $800 \mathrm{~W}$ on the scale of $20-100 \%$. The powder of Rhodiolae Radix was extracted in a Soxhlet apparatus with a mixture of chloroform-methanol $\left(2: 1,75^{\circ} \mathrm{C}\right)$, and pretreated with $80 \%$ ether twice to remove some coloured materials, oligosaccharides, and some small molecule materials. The organic solvent was volatilised and pretreated dry powder was obtained, as described previously (Gong et al., 2005; Li et al., 2009). Then the pretreated powder was extracted with distilled water in a microwave extraction apparatus at a selected extraction condition. After the extraction with microwave treatment, the extracts was centrifuged (2000 g, $20 \mathrm{~min}$ ), then the supernatant was separated from insoluble residue with nylon cloth (pore diameter: 38 um). The extracts were then defatted by the method of Sevag, precipitated by the addition of ethanol to a final concentration of $80 \%(\mathrm{v} / \mathrm{v})$, and the precipitates were collected by centrifugation (2000 g, $20 \mathrm{~min})$. It was then solubilised in deionised water and lyophilised to get polysaccharides from Rhodiolae Radix (PRR). The content of polysaccharides was determined by the phenol-sulphuric acid method at $490 \mathrm{~nm}$ (Li et al., 2009; Yang et al., 2008). Glucose was used as the standard and the standard curve is shown in Figure 1. The obtained linear regression equation is $A=0.0102 C+0.0205, R^{2}=0.9981$, suggesting that glucose concentration presented a good linear relation with absorbance in the range of 10 to $60 \mathrm{ug} / \mathrm{mL}$. The extraction yield of polysaccharides (\%) is calculated as the polysaccharides content of extraction divided by dried sample weight. 


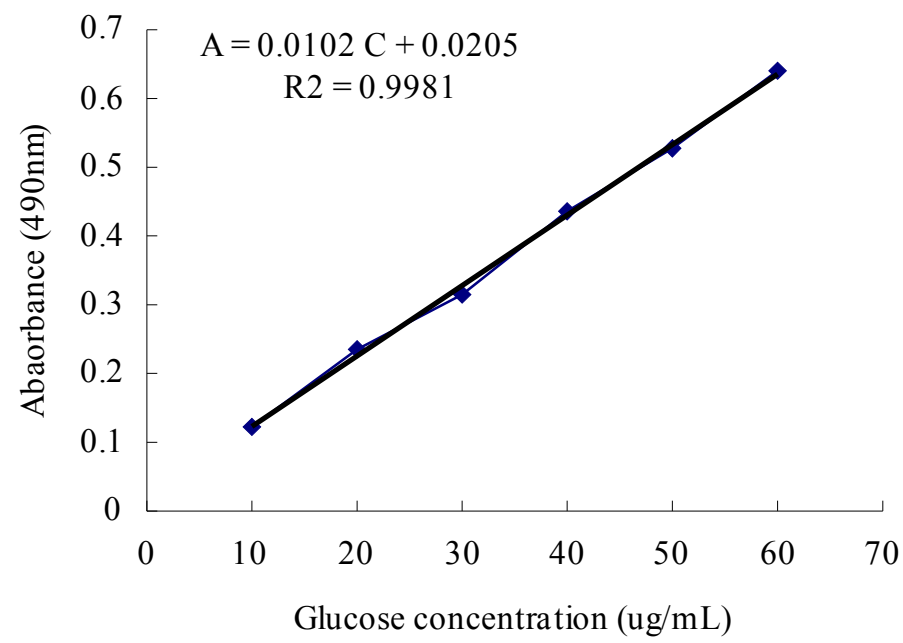

Figure 1: Standard curve of glucose

\section{Experimental design}

The experimental design for this study was divided into two major parts. Firstly, single factor experiments were performed to determine the appropriate range of MAE conditions of PRR. Three key factors influencing the extraction yield of PRR were investigated, including solid-liquid ratio, irradiation power and irradiation time. Secondly, the optimum MAE conditions of PRR were obtained by using orthogonal experiment design $\left(\mathrm{L}_{9}\left(3^{3}\right)\right)$ based on single factor experiments. In general, a full evaluation of the effect of three factors from three levels on the extraction yield of polysaccharides needs twenty-seven experiments. In order to reduce the number of experiments, orthogonal design was used, reducing the number of experiments to nine.

\section{Statistical analysis}

All the experiments were carried out in triplicate and the results were expressed by mean \pm SD. Software of orthogonal designing assistant II V3.1 was used for the evaluation of the statistical experimental design.

\section{Results and discussion}

\section{Effect of solid-liquid ratio on extraction yield of polysaccharides from Rhodiolae Radix}

In investigating the effect of solid-liquid ratio on extraction yield of PRR, several tests were performed at different solid-liquid ratio $(1: 10,1: 20,1: 30,1: 40,1: 50 \mathrm{~g} / \mathrm{mL})$. The rest of the extraction conditions were as follows: irradiation power of $480 \mathrm{~W}$ and irradiation time of $15 \mathrm{~min}$. As shown in Figure 2, with the increase of solid-liquid ratio from 1:10 to 1: $40 \mathrm{~g} / \mathrm{mL}$, the extraction yield of PRR quickly increased from 1.41 to $2.68 \%$. However, with the increase of the solid-liquid ratio from 1:40 to $1: 50 \mathrm{~g} / \mathrm{mL}$, extraction yield slowly increased from $2.68 \%$ to $2.76 \%$. Thus, $1: 40 \mathrm{~g} / \mathrm{mL}$ was chosen as the appropriate solid-liquid ratio. 


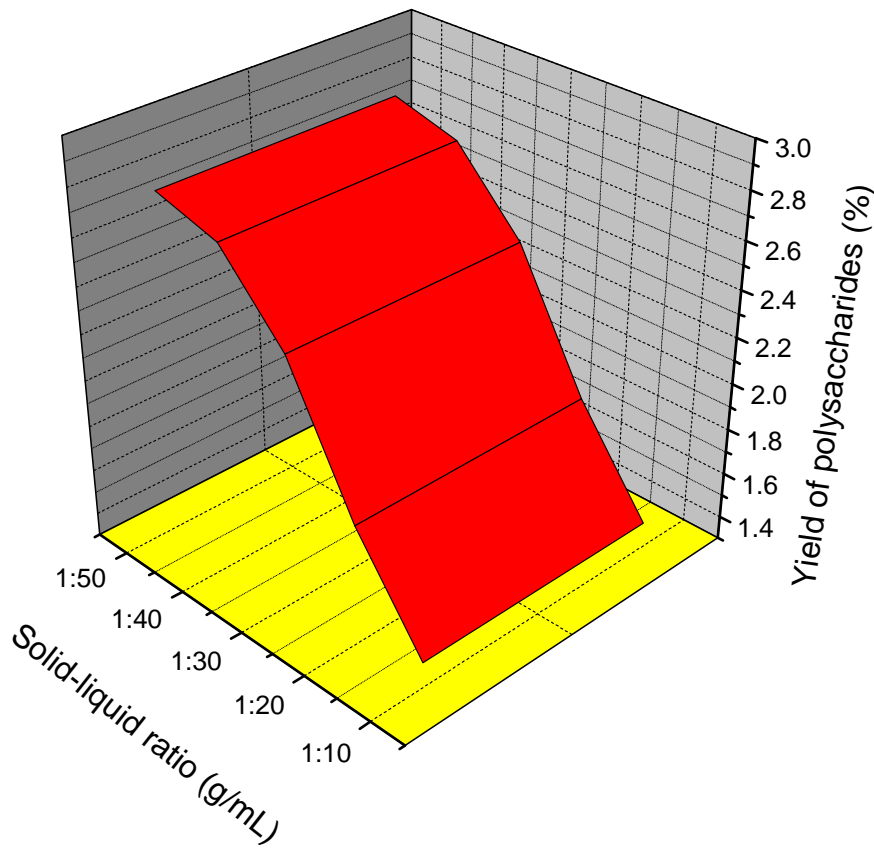

Figure 2: Effect of solid-liquid ratio on extraction yield of polysaccharides from Rhodiolae Radix

\section{Effect of irradiation power on extraction yield of polysaccharides from Rhodiolae Radix}

In investigating the effect of irradiation power on extraction yield of PRR, several tests were performed at different irradiation power $(160,320,480,640,800 \mathrm{~W})$. The rest of the extraction conditions were solid-liquid ratio of $1: 30 \mathrm{~g} / \mathrm{mL}$ and irradiation time of $15 \mathrm{~min}$. As shown in Figure 3, with the increase of irradiation power from 160 to $480 \mathrm{~W}$, the extraction yield of PRR quickly increased from 0.73 to $2.37 \%$. However, with the increase of the irradiation power from 480 to $800 \mathrm{~W}$, extraction yield quickly decreased from $2.37 \%$ to $1.36 \%$. Thus, $480 \mathrm{~W}$ was chosen as the appropriate irradiation power.

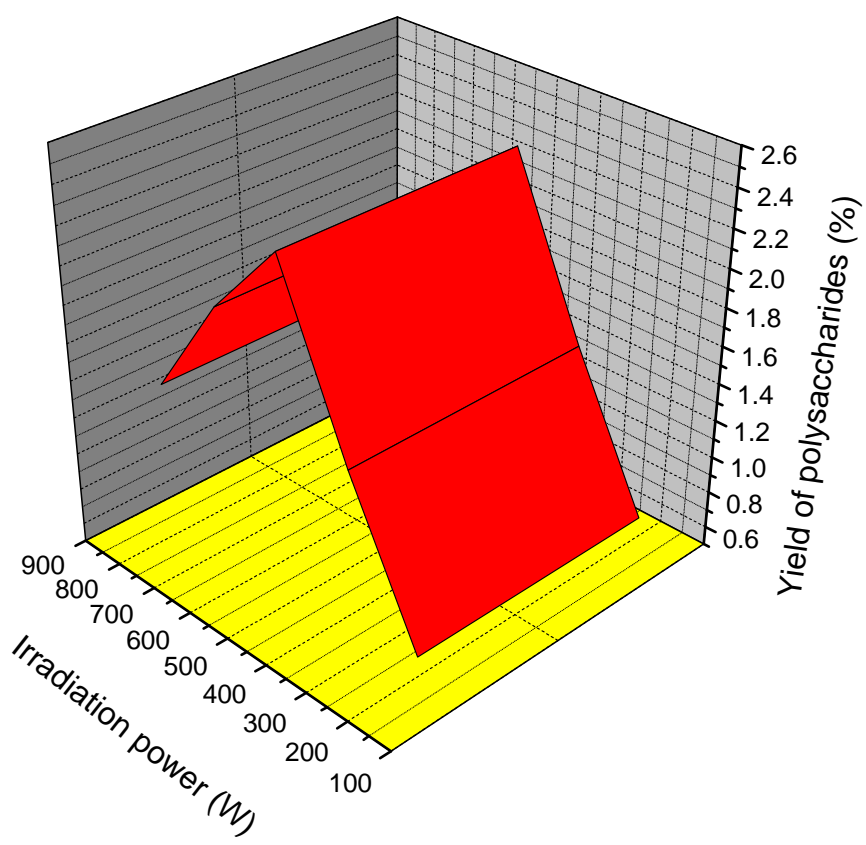

Figure 3: Effect of irradiation power on extraction yield of polysaccharides from Rhodiolae Radix 


\section{Effect of irradiation time on extraction yield of polysaccharides from Rhodiolae Radix}

In investigating the effect of irradiation time on extraction yield of PRR, several tests were performed at different irradiation time $(5,10,15,20,25 \mathrm{~min})$. The rest of the extraction conditions were solid-liquid ratio of $1: 30 \mathrm{~g} / \mathrm{mL}$ and irradiation power of $480 \mathrm{~W}$. As shown in Figure 4, with the increase of irradiation time from 5 to 10 min, the extraction yield of PRR quickly increased from 1.09 to $2.84 \%$. However, with the increase of the irradiation time from 10 to $25 \mathrm{~min}$, extraction yield decreased from $2.84 \%$ to $1.85 \%$. Thus, 10 min was chosen as the appropriate irradiation time.

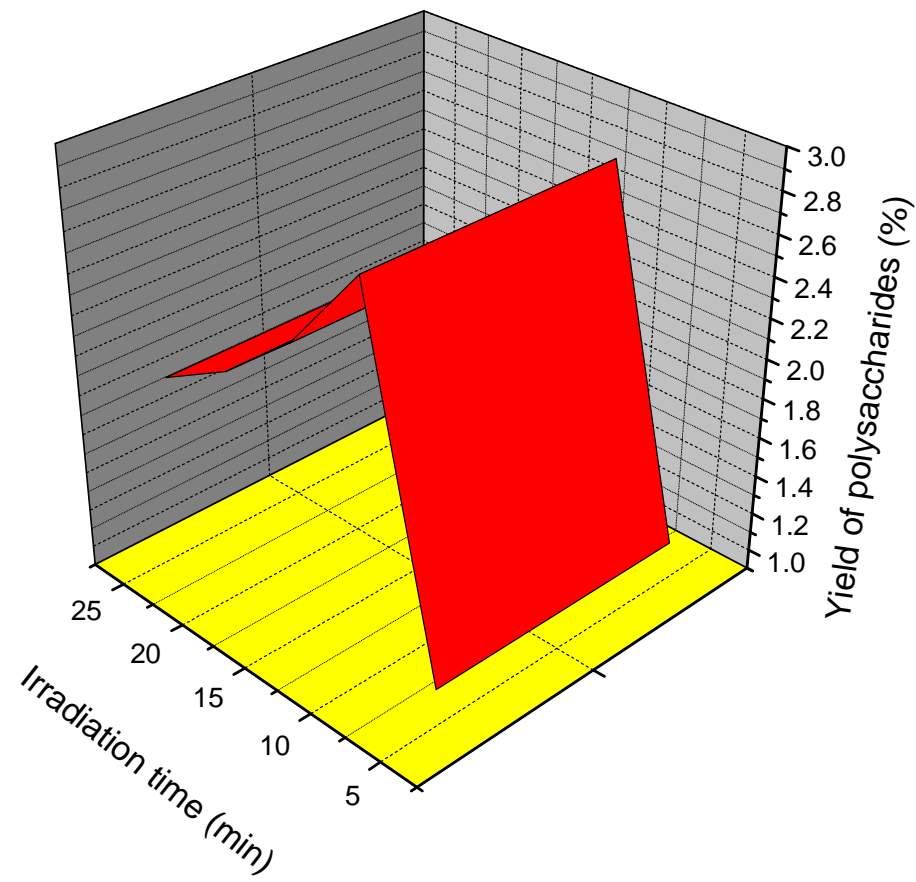

Figure 4: Effect of irradiation power on extraction yield of polysaccharides from Rhodiolae Radix

\section{Orthogonal experiment of microwave-assisted extraction of polysaccharides from Rhodiolae Radix}

Since various factors potentially affect the MAE process, the optimum extraction conditions represent a critical step in the development of an MAE method. In this study, integrating the results from the single factor experiments, three key factors influencing the polysaccharide yield were selected, thus: solid-liquid ratio, irradiation power and irradiation time. Subsequently, a three-factor and three-level orthogonal experiment (Table 1) was designed according to the $\mathrm{L}_{9}\left(3^{3}\right)$ table. The results are shown in Table 2.

According to the value of range $\mathrm{R}$ in Table 2, irradiation power (factor $\mathrm{B}$ ) exerted the most significant effect on extraction yield of PRR, and the order of importance that influenced extraction yield of PRR was found to be $\mathrm{B}$ (irradiation power) $>\mathrm{C}$ (irradiation time) $>\mathrm{A}$ (solid-liquid ratio). The optimum MAE conditions of PRR were A3B2C1, namely, solid-liquid ratio of $1: 45 \mathrm{~g} / \mathrm{mL}$, irradiation power of $480 \mathrm{~W}$, and irradiation time of 8 min, while extraction yield of PRR was $3.24 \%$.

\section{Conclusion}

An efficient method has been developed for the extraction of polysaccharides from Rhodiolae Radix. The optimised microwave-assisted extraction conditions were as follows: solid-liquid ratio of 1:45 g/mL, irradiation power of $480 \mathrm{~W}$ and irradiation time of $8 \mathrm{~min}$, while extraction yield of polysaccharides was $3.24 \%$. Microwave-assisted extraction method has a great potential for large-scale extraction of polysaccharides from Rhodiolae Radix. It might also be useful for the extraction 
http://dx.doi.org/10.4314/ajtcam.v10i4.29

of other biologically active compounds from different tissues of plant materials.

Table 1: Three-factor and three-level of orthogonal experimental

\begin{tabular}{|l|l|l|l|}
\hline \multirow{2}{*}{ Level } & \multicolumn{2}{|l|}{ Factor } & \multicolumn{2}{|l|}{} \\
\cline { 2 - 4 } & $\begin{array}{l}\text { A (solid-liquid ratio, } \\
\mathrm{g} / \mathrm{mL})\end{array}$ & B (irradiation power, W) & C (irradiation time, min) \\
\hline 1 & $1: 35$ & 320 & 8 \\
\hline 2 & $1: 40$ & 480 & 10 \\
\hline 3 & $1: 45$ & 640 & 12 \\
\hline
\end{tabular}

Table 2: Results of orthogonal experimental

\begin{tabular}{|l|l|l|l|l|}
\hline Test Number & $\begin{array}{l}\text { A (solid-liquid ratio, } \\
\mathrm{g} / \mathrm{mL})\end{array}$ & $\begin{array}{l}\text { B (irradiation power, } \\
\mathrm{W})\end{array}$ & $\begin{array}{l}\mathrm{C} \quad \text { (irradiation } \\
\text { time, } \mathrm{min})\end{array}$ & $\begin{array}{l}\text { Yield of polysaccharides } \\
(\%)\end{array}$ \\
\hline 1 & 1 & 1 & 1 & 1.87 \\
\hline 2 & 1 & 2 & 2 & 2.91 \\
\hline 3 & 1 & 3 & 3 & 2.27 \\
\hline 4 & 2 & 1 & 2 & 1.63 \\
\hline 5 & 2 & 2 & 3 & 2.69 \\
\hline 6 & 2 & 3 & 1 & 2.38 \\
\hline 7 & 3 & 1 & 3 & 1.76 \\
\hline 8 & 3 & 2 & 1 & 3.24 \\
\hline 9 & 3 & 3 & 2 & 2.21 \\
\hline $\mathrm{X} 1$ & 2.350 & 1.753 & 2.497 & \\
\hline $\mathrm{X} 2$ & 2.233 & 2.947 & 2.250 & \\
\hline $\mathrm{X} 3$ & 2.403 & 2.287 & 2.240 & \\
\hline $\mathrm{R}$ & 0.170 & 1.194 & 0.257 & \\
\hline
\end{tabular}

\section{Acknowledgements}

This work was supported by the Science and Technology Development Foundation of Jilin Province of China (Grant No. 20120918) and Reserve Foundation of Jilin Agricultural Science and Technology College (Grant No. 2012-409).

\section{References}

1. Cheng, X.J., Di, L., Wu, Y., Zhao, Q.C., Du, G.Z., and Liu, Y.Q. (1993). Studies on the hypoglycemic effect of Rhodiola sachalinensis A. Bor. polysaccharides. Zhongguo Zhong Yao Za Zhi 18: 557-559.

2. Choe, K.I., Kwon, J.H., Park, K.H., Oh, M.H., Kim, M.H., Kim, H.H., Cho, S.H., Chung, E.K., Ha, S.Y., and Lee, M.W. (2012). The Antioxidant and Anti-inflammatory Effects of Phenolic Compounds Isolated from the Root of Rhodiola sachalinensis A. BOR. Molecules 17:11484-11494.

3. Gong, G.M., Wang, H.T., Han, N. (2005). Extraction of Polysccharide from Rhodiola 1. with Ultrasonic Wave. Food Sci 26: 127-130. 
4. Li, F., Li, Q., Gao, D., Peng, Y., and Feng, C. (2009). Preparation and antidiabetic activity of polysaccharide from Portulaca oleracea L. Afri J Biotech 8: 569-573.

5. Li, F., Tang, H., Xiao, F., Gong, J., Peng, Y., and Meng, X. (2011). Protective effect of salidroside from Rhodiolae Radix on diabetes-induced oxidative stress in mice. Molecules 16: 9912-9924.

6. Li, J., Zhu, B., Chen, Y., and Chen, Z. (2006). Effect of Rhodiola Polysaccharide on Peripheral Blood Cells and Bone Marrow Cell Cycle of myelosuppressed Anemia Mice. Sichuan Journal of Anatomy 14: 4-7.

7. Mao, Y., Li, Y., and Yao, N. (2007). Simultaneous determination of salidroside and tyrosol in extracts of Rhodiola L. by microwave assisted extraction and high-performance liquid chromatography. J Pharm Biomed Anal 45: 510-515.

8. Piao, H., Li, Y., Li, H., Wei, C., and Zheng, C. (2000). Immunoregulatory effects of Rhodiola Sachinensis A. Bor Polysaccarides In mice. J Med Sci Yanbian Univ 4: 251-254.

9. Romanik, G., Gilgenast, E., Przyjazny, A., and Kamiński, M. (2007). Techniques of preparing plant material for chromatographic separation and analysis. J Biochem Biophys Methods 70: 253-261.

10. Su, X.F., Zhang, H., Shao, J.X., and Wu, H.Y. (2007). Theoretical study on the structure and properties of crenulatin molecule in herb Rhodiola crenulata. J Mol Struct THEOCHEM 847: 59-67.

11. Wang, J., Zhang, J., Wang, X., Zhao, B., Wu, Y., and Yao, J. (2009). A comparison study on microwave-assisted extraction of Artemisia sphaerocephala polysaccharides with conventional method: Molecule structure and antioxidant activities evaluation. Int J Biol Macromol 5: 483-492.

12. Wiedenfeld, H., Dumaa, M., Malinowski, M., Furmanowa, M., and Narantuya, S. (2007). Phytochemical and analytical studies of extracts from Rhodiola rosea and Rhodiola quadrifida. Pharmazie 62: 308-311.

13. Wu, J., Lin, L., and Chau, F.T. (2001). Ultrasound-assisted extraction of ginseng saponins from ginseng roots and cultured ginseng cells. Ultrason Sonochem 8: 347-352.

14. Wu, S., Zu, Y., and Wu, M. (2003). High yield production of salidroside in the suspension culture of Rhodiola sachalinensis. J Biotechnol 106: 33-43.

15. Xie, J.H., Xie, M.Y., Shen, M.Y., Nie, S.P., Li, C., and Wang, Y.X. (2010). Optimisation of microwave-assisted extraction of polysaccharides from Cyclocarya paliurus (Batal.) Iljinskaja using response surface methodology. J Sci Food Agric 90: $1353-1360$.

16. Yan, X., Wu, S., Wang, Y., Shang, X., and Dai, S. (2004). Soil nutrient factors related to salidroside production of Rhodiola sachalinensis distributed in Chang Bai Mountain. Environ Exp Bot 52: 267-276.

17. Yang, B., Zhao, M., Shi, J., Yang, N., and Jiang, Y. (2008). Effect of ultrasonic treatment on the recovery and DPPH radical scavenging activity of polysaccharides from longan fruit pericarp. Food Chem 106: 685-690.

18. Zhang, S., Bi, H., and Liu, C. (2007). Extraction of bio-active components from Rhodiola sachalinensis under ultrahigh hydrostatic pressure. Sep Purif Technol 57:277-282.

19. Zhang, Y., and Liu, X. (2009). Anti-Coxsackievirus B3 effects of Rhodiola Sachalinensis polysaccaride in vitro. Chin J Hospital Pharmacy 20: 16-18. 